\title{
CONCORDANCIA ENTRE LA ESTIMACIÓN VISUAL Y LA MEDICIÓN DEL VOLUMEN RECOLECTADO EN UNA BOLSA DEL SANGRADO INTRAPARTO EN MUJERES CON PARTO NORMAL EN BOGOTÁ, COLOMBIA, 2006
}

Agreement between visual estimation and the objective determination of the volume of intrapartum blood loss in a collecting bag in women undergoing vaginal delivery. Bogotá, Colombia, 2006

Jorge A. Rubio-Romero, M.D. *, Hernando G. Gaitán-Duarte, M.D.**, Nelcy Rodríguez-Malagón, M.D. ***

Recibido: febrero 18/08 - Aceptado: mayo 12/08

\section{RESUMEN}

Objetivos: establecer la concordancia entre la estimación visual y la medición del volumen recolectado en una bolsa del sangrado intraparto, según las características del personal encargado de la atención del parto en un hospital universitario de mediana complejidad y la correlación entre el volumen recolectado y el cambio en la hemoglobina y el hematocrito posparto.

Materiales y métodos: se realizó un estudio de concordancia en una cohorte de 168 embarazadas atendidas por parto normal, en un hospital general

\footnotetext{
Profesor Asociado. Departamento de Obstetricia y Ginecología. Universidad Nacional de Colombia. Bogotá, Colombia.

Correo electrónico: jarubior@unal.edu.co

* Profesor titular. Departamento de Obstetricia y Ginecología. Instituto de Investigaciones Clínicas. Universidad Nacional de Colombia. Bogotá, Colombia.

*** Profesora Asociada. Departamento de Estadística. Instituto de Investigaciones Clínicas. Universidad Nacional de Colombia. Bogotá, Colombia
}

de nivel medio de complejidad ubicado en Bogotá, Colombia. Se evaluó la concordancia por observadores simultáneos de niveles crecientes de formación y experiencia, mediante el coeficiente de correlaciónconcordancia (CCC Lin), límites de acuerdo del 95\% y la correlación entre el cambio en la hemoglobina y hematocrito con el volumen recolectado mediante el coeficiente de Pearson.

Resultados: la mediana del volumen recolectado fue $494 \mathrm{~mL}$ (p5 = 110, p95 = 1.320), la de la caída del hematocrito de 3,8\% y de hemoglobina de 1,2 $\mathrm{g} / \mathrm{dL}$. La concordancia global fue aceptable [CCC $=0,72$ IC 95\%: 0,67-0,76)] con una subestimación visual media de 110,2 mL (límites de acuerdo 95\% $=-562,1 \mathrm{~mL}$ y $341,8 \mathrm{~mL}$ ). La subestimación y la pérdida de la confiabilidad fueron crecientes a mayor volumen recolectado para todas las categorías de evaluadores. La correlación entre volumen recolectado y el cambio en hemoglobina y hematocrito fue 0,55 y 0,53 . 
Conclusiones: la confiabilidad de la estimación visual es aceptable para volúmenes pequeños y disminuye al aumentar el volumen recolectado. Se deben introducir instrumentos y educación continuada que permitan mejorar la confiabilidad de la estimación del sangrado intraparto para prevenir la hemorragia posparto.

Palabras clave: hemorragia posparto, percepción visual, reproducibilidad de resultados, confiabilidad.

\section{SUMMARY}

Objective: to establish agreement between visual estimation and volume of blood collected in a bag during normal delivery according to the different observer characteristics in a medium complexity teaching hospital and the correlation between the collected volume and mean change in haemoglobin and haematocrite.

Methods: a concordance study was carried out, comparing visual estimation by simultaneous observers having various degrees of education and experience to the objective measurement of the volume collected in a sterilised bag during and until the first hour post partum. The study included 168 pregnant women who underwent normal vaginal delivery. Concordance was evaluated by concordance correlation coefficient (CCC Lin), 95\% limits of agreement and Pearson's correlation coefficient for changes in haemoglobin concentration and haematocrite with measured collected volume.

Results: median collected volume during normal delivery was 494 millilitres $(\mathrm{mL})(\mathrm{p} 5=110$, p95 = 1320), median change of haematocrite 3,8\% and haemoglobin 1,2 $\mathrm{g} / \mathrm{dL}$. Global concordance was acceptable [CCC $=0.72$ (IC95\%: 0.67-0.76) ] with an average visual underestimation of 110,2 $\mathrm{mL}$ (limits of agreement: $-562.1 \mathrm{~mL}$ to $341.8 \mathrm{~mL}$ ). Underestimation and loss of reliability increased with the greater volumes collected for all of the observers' categories. Correlations between collected volume and change in haemoglobin and haematocrite were 0.55 and 0.53 respectively.
Conclusions: the reliability of visual estimation was acceptable for the small volumes collected and decreased as collected volume increased. Introducing measuring instruments and continued education is necessary for improving visual estimation reliability to prevent post partum haemorrhage.

Key words: post-partum haemorrhage, visual perception, reproducibility of results, reliability.

\section{INTRODUCCIÓN}

Las complicaciones hemorrágicas durante el embarazo o el parto representan la tercera causa de la morbilidad y mortalidad materna en el país ${ }^{1}$ y la primera causa de las 529.000 muertes maternas que se calcula ocurren cada año en el mundo, de estas el 99\% ocurre en los países en desarrollo y el 95\% se atribuye a causas prevenibles durante el control médico. ${ }^{2}$

La muerte de mujeres por hemorragia asociada al embarazo, al parto y al puerperio viene en descenso desde hace varios años en Colombia. Estudios nacionales mostraron que la hemorragia fue la causa del 57\% de muertes maternas en Cali entre 1985 y 1994, el 38\% de las muertes maternas reportadas en Bogotá en 1995 y 1996 y el 28\% de las que se presentaron en Medellín en el año 2005. -6 $^{3-6}$ Es importante resaltar que el $60 \%$ de las muertes por hemorragia ocurren en partos vaginales y en el 73,2\% la atención inicial se realizó en los niveles bajos de complejidad. ${ }^{6}$

El sangrado es un fenómeno fisiológico inherente al parto y al puerperio. La cuantificación del sangrado intraparto es un reto clínico y existe una gran divergencia entre los valores considerados "normales" durante la atención de partos de bajo riesgo.? Mediante una técnica de dilución de hemoglobina, Wallace estimó la magnitud del sangrado en cerca de $450 \mathrm{~mL}$ y consideró que el 6\% de las mujeres presentaron un sangrado mayor de $500 \mathrm{~mL} .^{8}$ Estudios más recientes, con mediciones del nivel de hemoglobina seriadas y recolección en bolsas del sangrado intraparto, han permitido estimar que el sangrado normal o usual durante el parto varía entre 
150 y $600 \mathrm{~mL}$ en las primeras 24 horas. ${ }^{9-11}$ La variabilidad en el volumen del sangrado ha dificultado tener una válida estimación del momento en el que la magnitud del sangrado deja de ser "normal" y se convierte en una hemorragia anormal intraparto, que motiva iniciar un tratamiento inmediato que prevenga la morbilidad y la mortalidad asociada.

El problema clínico radica en que frecuentemente solo cuando llega la descompensación hemodinámica se determina el diagnóstico de hemorragia posparto y se inicia el tratamiento. La rapidez con la que una gestante progresa desde un sangrado leve, compensado fisiológicamente y, por lo tanto, considerado "normal", hacia un estado de descompensación hemodinámica, en ausencia de otros signos clínicos predominantes, hace muy difícil determinar el momento crítico del diagnóstico, aun mientras la paciente se vigila muy cerca.

La norma de atención del parto, contenida en la Resolución 412 del año 2000 del Ministerio de Protección Social de la República de Colombia, ${ }^{12}$ recomienda la estimación visual y la determinación clínica del sangrado, para establecer el diagnóstico de hemorragia anormal del posparto. Sin embargo pocos estudios han evaluado la validez y confiabilidad de la estimación visual.

La confiabilidad de la estimación visual del sangrado dependería del entrenamiento y experiencia del personal de salud que realiza la atención del parto, sin embargo, se ha informado que los años de experiencia no incrementan la capacidad de estimar el sangrado y la tendencia a sobreestimar los sangrados pequeños y subestimar la magnitud de los sangrados importantes. Por otra, parte se demostró que un programa de entrenamiento lleva a una mejora significativa de la estimación del sangrado. ${ }^{13}$

Se ha descrito como alternativa el uso de una bolsa recolectora durante el parto, como un método rápido y preciso para el diagnóstico de hemorragia posparto que permite tanto al personal con más experiencia como al personal en entrenamiento evaluar objetivamente el sangrado, mejorar sus habilidades para la estimación visual y aumentar la precisión diagnóstica de la magnitud del sangrado. ${ }^{14}$ No se ha evaluado la concordancia entre la estimación visual y el volumen contenido en la bolsa recolectora.

Así pues, la estimación visual de la cantidad de hemorragia resulta ser imprecisa y poco confiable aun en manos expertas. Es necesario evaluar alternativas que mediante la estimación clínica visual permitan determinar la magnitud del sangrado durante el parto y seleccionar las más efectivas y confiables de tal manera que permitan acercarse al diagnóstico de hemorragia anormal en forma más temprana, a menor costo y con menor riesgo de complicaciones. Por lo tanto, el objetivo del presente estudio fue evaluar la confiabilidad de la estimación visual con la medición objetiva del volumen contenido en ella y la correlación entre el volumen recolectado con los métodos de estimación indirectos (cambio en el hematocrito y en la concentración de hemoglobina) para el diagnóstico de la hemorragia anormal posparto.

\section{MATERIALES Y MÉTODOS}

Se realizó un estudio de concordancia diagnóstica, ensamblado en una cohorte conformada por mujeres con embarazos viables en trabajo de parto en el hospital de Engativá ESE II nivel (hospital de nivel medio de complejidad), donde se atienden pacientes de todos los regímenes de aseguramiento del sistema general de seguridad social en salud y donde realizan prácticas estudiantes de medicina de la Universidad Nacional de Colombia. El protocolo cumplió con los requerimientos contemplados en la Declaración de Helsinki y la Resolución 8430 del Ministerio de Salud de Colombia y fue aprobado por los comités de ética e investigaciones de la Facultad de Medicina y el hospital de Engativá.

Población: se incluyeron mujeres mayores de 16 años que cursaban con gestaciones de bajo riesgo con fetos viables en presentación cefálica que ingresaron para atención de parto vaginal y aceptaron participar en el estudio, firmaron el consentimiento informado y fueron atendidas por 
estudiantes de pre y posgrado, bajo la supervisión de personal docente o institucional. Se excluyeron las mujeres con atención de parto por cesárea por cualquier indicación obstétrica, parto en pelvis y embarazos gemelares. Así mismo, las mujeres en las que no se dispuso del resultado de la hemoglobina y el hematocrito antes del parto, el peso de la bolsa recolectora o el resultado de la hemoglobina y el hematocrito en las primeras 24 horas de ocurrido el parto. Se realizó un muestreo consecutivo de las pacientes atendidas hasta completar el tamaño de muestra programado, calculado según una hipótesis a una cola, confiabilidad del $95 \%$ y poder del $90 \%$, de un valor de correlación-concordancia de $\operatorname{Lin}^{15,16}$ mínimo esperado de 0,5 entre la estimación visual y el volumen recolectado para lo que se requerirían 52 mediciones por método. Esperando un mínimo de correlación de $(\mathrm{r})=0,3$ a una cola entre el volumen recolectado y el cambio en la hemoglobina, se requerirían 113 pacientes. Anticipando un 10\% de pérdidas en el seguimiento y hasta un $10 \%$ de errores en la medición, se calculó una muestra final de 160 pacientes.

Procedimiento: una vez firmado el consentimiento informado, se verificó la hemoglobina y hematocrito previo al parto. Se condujo el trabajo de parto según las guías y protocolos institucionales. Durante el expulsivo, con la paciente en posición de litotomía, se evacuó la vejiga por micción espontánea y la ampolla rectal mediante masaje de la pared vaginal posterior para disminuir la caída de orina y heces dentro de la bolsa recolectora. Se realizó aseo genital previo a la colocación de los campos estériles y amniotomía en caso de que esta no hubiera ocurrido durante el trabajo de parto. Luego se colocó una bolsa roja, opaca, de 120 por 100 centímetros, calibre 3, estéril, bajo la cadera y glúteos de la paciente para recolectar el sangrado ocurrido a partir de entonces. Esta bolsa permaneció durante la atención del parto, el alumbramiento y la sutura perineal cuando esta fue necesaria hasta que la paciente se trasladó a recuperación, antes de la primera hora posparto. Terminada la atención del parto, quien lo atendió calculó el volumen de líquido recolectado dentro de la bolsa. Simultáneamente, un segundo observador realizó la estimación visual del volumen contenido en la bolsa durante este mismo periodo, de manera independiente y ciega a la estimación del primer observador. Finalmente, la bolsa se retiró, selló y pesó en una báscula electrónica (Lexus ${ }^{\circledR}$ modelo Fénix de 6 kilogramos de capacidad y precisión de 0,5 gramos) debidamente calibrada. Se estimó un volumen de un centímetro cúbico por cada gramo contenido en la bolsa, una vez restado el peso seco de la bolsa y del recipiente. El resultado se mantuvo oculto a los observadores al igual que el peso del recipiente y la bolsa en seco. El papel de supervisor siempre lo realizó personal con mayor nivel de formación que el operador. En caso de sangrado anormal del posparto, la bolsa permaneció en su sitio hasta que la paciente fue estabilizada o trasladada fuera de la sala de parto, sin exceder el tiempo de una hora. Se midió la concentración de hemoglobina y el hematocrito a las doce horas de ocurrido el nacimiento a todas las pacientes incluidas en el estudio y se les realizó seguimiento durante su internación y hasta la primera semana del parto para identificar las complicaciones inmediatas, la necesidad de transfusiones, taponamiento uterino o presencia de sangrado anormal en el puerperio tardío.

Variables de desenlace: se estableció como hemorragia posparto cuando se realizó este diagnóstico en la historia clínica o se reportó el uso de maniobras médicas o quirúrgicas para controlar el sangrado o la transfusión de glóbulos rojos empaquetados o sangre total. También se consideró hemorragia posparto si el cambio (resta de los valores basales y los tomados a las 12 horas posparto) del porcentaje del hematocrito fue mayor o igual a $10 \%$.

Análisis: los datos se analizaron mediante el paquete estadístico Stata S/E C) versión 10.0 (StatCorp@ 1987-2007) para Windows $®$. Se hizo una descripción de las características demográficas de las pacientes atendidas y los observadores con medidas 
de tendencia central y dispersión para las variables continuas, y proporciones para las variables nominales y categóricas.

El análisis de concordancia se realizó mediante el coeficiente de correlación-concordancia de Lin (CCC). ${ }^{14-16}$ El CCC permite establecer si los datos obtenidos se desvían de manera significativa de la línea de concordancia perfecta (intercepto en el origen y pendiente de uno a 45 grados) siendo robusto en distribuciones asimétricas con tamaños de muestra mayores de 100 individuos. ${ }^{17} \mathrm{El} \mathrm{CCC}$ comprende dos componentes: el primero es el sesgo o medida de exactitud de los datos obtenidos por el instrumento, es decir, la cercanía de los datos a la línea de concordancia. El segundo es la precisión, o sea, la distancia o dispersión de los datos obtenidos alrededor de la línea de mejor ajuste de los datos obtenidos. La precisión corresponde al coeficiente de correlación de Pearson. ${ }^{18}$ En caso de un acuerdo perfecto (precisión y exactitud) el CCC adquiere el valor de 1 . Se utilizaron los valores propuestos por Fleiss y colaboradores ${ }^{19}$ para la valoración e interpretación de la concordancia: mayor de 0,90 muy buena, entre 0,71 y 0,90 aceptable, entre 0,510,70 moderada, entre 0,31-0,50 mediocre y menor de 0,30 nula. Se realizaron gráficas de dispersión entre el valor estimado y el volumen recolectado en la bolsa, se estimó la línea de regresión de los datos obtenidos junto a la línea de concordancia perfecta para representar gráficamente el significado del CCC y se graficaron las diferencias entre los valores obtenidos por cada método contra la media de los valores encontrados por los métodos para cada paciente evaluado, para determinar los límites de acuerdo de Bland y Altman. ${ }^{20-23}$ Estos límites representan los valores dentro de los que se encuentran el 95\% de las diferencias entre las mediciones obtenidas por cada método. Esta aproximación permite evaluar la magnitud de las diferencias en un contexto clínico y estimar la variabilidad entre ambas mediciones.

La correlación entre el valor recolectado de sangrado y el cambio en la concentración de la he- moglobina y el hematocrito se evaluó mediante el coeficiente de correlación de Pearson.

\section{RESULTADOS}

Durante el periodo comprendido entre noviembre $1^{\circ}$ del 2006 y marzo 31 del 2007, 1.105 mujeres ingresaron al hospital de Engativá ESE II nivel para la atención de parto vaginal de bajo riesgo. 120 fueron excluidas por ser menores de 16 años de edad y de las 985 restantes 199 aceptaron participar en el estudio firmando el consentimiento informado.

Se incluyeron 168 pacientes para el análisis, ya que de las 199 pacientes participantes, tres (3) fueron excluidas porque requirieron de cesárea durante el trabajo de parto, cuatro (4) pacientes fueron excluidas del estudio debido a que la bolsa recolectora fue desechada sin ser pesada, y veinticuatro (24) se excluyeron del análisis porque fueron dadas de alta sin la toma de muestra para hematocrito y hemoglobina a las 12 horas posparto. Estas pacientes se siguieron entre el $7^{\circ}$ y el 10 día posparto para descartar la presencia de anemia, infección puerperal o sangrado anormal del puerperio. Ninguna de ellas presentó disminución del hematocrito mayor del 10\% que hiciera pensar en hemorragia posparto no detectada.

En cuanto a los observadores se contó con la participación voluntaria de 20 médicos especialistas, 14 residentes y 48 estudiantes de medicina. Un médico especialista reportó haber recibido educación en estimación visual del sangrado. La experiencia de los médicos con título de especialista desde el momento del grado como médico estuvo entre 4 y 28 años, con promedio de 13 años; y la de los médicos residentes estuvo entre nueve meses y seis años, con promedio de dos años y medio.

La tabla 1 muestra las características demográficas de las pacientes reclutadas y de la atención del parto. Veinte pacientes (11,9\%) tuvieron hemoglobina menor de $12 \mathrm{~g} / \mathrm{dl}$, siendo consideradas anémicas para la altura sobre el nivel del mar de la ciudad de Bogotá. ${ }^{24}$ Se practicó amniotomía al $80,2 \%$ de las pacientes con membranas íntegras. 
Tabla 1. Características demográficas de las pacientes y de la atención del parto.

\begin{tabular}{|c|c|}
\hline Característica & Valor \\
\hline Promedio de edad (rango) & 23,9 años (17-42) \\
\hline $\begin{array}{l}\text { Mediana edad gestacional } \\
\text { (rango) }\end{array}$ & 39 semanas $(32-42)$ \\
\hline Nulíparas (\%) & $68(40,5)$ \\
\hline Antecedente de aborto (\%) & $32(19,1)$ \\
\hline $\begin{array}{l}\text { Ausencia de control prenatal } \\
\text { (\%) }\end{array}$ & $5(3)$ \\
\hline $\begin{array}{l}\text { Presencia de cuadro } \\
\text { hemático III trimestre (\%) }\end{array}$ & $103(61,1)$ \\
\hline $\begin{array}{l}\text { Promedio hemoglobina } \\
\text { anteparto (rango) }\end{array}$ & $13,2 \mathrm{~g} / \mathrm{dL}(10,3-16)$ \\
\hline $\begin{array}{l}\text { Promedio hematocrito } \\
\text { anteparto (rango) }\end{array}$ & $38,8 \%(30,4-49,2)$ \\
\hline $\begin{array}{l}\text { Antecedente de ruptura de } \\
\text { membranas anteparto (\%) }\end{array}$ & $35(20,8)$ \\
\hline $\begin{array}{l}\text { Inducción del trabajo de } \\
\text { parto (\%) }\end{array}$ & $35(20,8)$ \\
\hline Refuerzo con oxitocina (\%) & $85(50,6)$ \\
\hline Uso de episiotomía (\%) & $21(12,5)$ \\
\hline Parto instrumentado (\%) & $5(3)$ \\
\hline $\begin{array}{l}\text { Mediana duración ruptura de } \\
\text { membranas (rango) }\end{array}$ & 120 minutos $(2-1.520)$ \\
\hline $\begin{array}{l}\text { Mediana duración expulsivo } \\
\text { (rango) }\end{array}$ & 28,5 minutos $(3-178)$ \\
\hline $\begin{array}{l}\text { Mediana duración del } \\
\text { alumbramiento (rango) }\end{array}$ & 3 minutos $(0-23)$ \\
\hline $\begin{array}{l}\text { Mediana duración de uso de } \\
\text { la bolsa (rango) }\end{array}$ & 21 minutos $(7-74)$ \\
\hline
\end{tabular}

Ninguna paciente recibió analgesia peridural para la conducción ni para la atención del parto. Solo 35 pacientes $(20,8 \%)$ recibieron en forma completa las tres maniobras descritas para el manejo activo del alumbramiento. La mediana de duración del alumbramiento fue de tres (3) minutos. El peso promedio de los recién nacidos fue de 3.099 gramos, en un rango entre 1.730 y 4.150 gramos. La proporción de recién nacidos de bajo peso fue de 5,36\%. El volumen estimado, el recolectado en la bolsa, y los cambios en la hemoglobina y el hematocrito durante el parto se muestran en la tabla 2. En 10 (5,9\%) de las 168 pacientes, el médico tratante inició manejo para hemorragia anormal intraparto, y durante el seguimiento se detectaron nueve casos $(5,4 \%)$ con disminución mayor del 10\% del hematocrito. Solo uno de los casos que recibió tratamiento para controlar la hemorragia presentó un descenso mayor al 10\% del hematocrito. Sumados estos casos, la incidencia de hemorragia posparto encontrada en el presente estudio fue 18/168 que equivale al 10,7\%. La causa del $66,6 \%$ de las hemorragias posparto correspondió a atonía uterina y el 33,3\% restante a retención de restos placentarios.

La concordancia calculada mediante el CCC de Lin $(\rho \mathbb{C})$ entre la estimación visual y el volumen recolectado dentro de la bolsa fue de 0,72 (IC 95\% 0,67-0,76). Los componentes del coeficiente fueron: precisión de 0,79 , factor de corrección de sesgo (exactitud) de 0,91 y pendiente de 1,33. La diferencia promedio entre ambos métodos (visual y volumen recolectado) fue de $-110,2 \mathrm{~mL}$, con límites de acuerdo del 95\% entre -562,1 y 341,8 mL (rango $=903,8 \mathrm{~mL}$ ). El análisis gráfico de los componentes del CCC y los límites de acuerdo se muestran en la figura 1.

Las tablas 3 y 4 muestran los componentes del CCC y los límites de acuerdo del 95\% según el papel desempeñado por los observadores durante la atención del parto, su nivel de formación y sus años de experiencia. No se observaron diferencias significativas entre los grupos evaluados.

La correlación encontrada entre el cambio en la concentración de hemoglobina, el cambio del hematocrito y el volumen recolectado dentro de la bolsa fue de 0,55 y 0,53 respectivamente.

\section{DISCUSIÓN}

La estimación visual durante la atención del parto es la herramienta de primera mano para el inicio de las conductas clínicas que permitan controlar el sangrado excesivo anormal y evitar el deterioro de la salud de las gestantes en el puerperio inmediato. 
Tabla 2. Distribución del sangrado estimado y recolectado en la bolsa, durante la atención del parto vaginal, y correlación con el cambio en la hemoglobina y hematocrito.

\begin{tabular}{|l|l|l|l|l|l|l|l|l|}
\hline & $\mathbf{9}$ & \multicolumn{7}{|c|}{ Percentiles } \\
\hline Volumen de sangrado estimado $\mathbf{( m L )}$ & 80 & 130 & 240 & 360 & 570 & 800 & 990 & 431,2 \\
\hline Volumen de sangrado recolectado (mL) & 110 & 163 & 296 & 454 & 715 & 1.035 & 1320 & 541,4 \\
\hline Diferencia hemoglobina (gramos/dL) & $-0,6$ & $-0,1$ & 0,5 & 1,2 & 1,9 & 2,8 & 3,4 & 1,2 \\
\hline Diferencia hematocrito (\%) & $-0,9$ & 0,3 & 1,8 & 3,8 & 5,9 & 8,3 & 10 & 4,1 \\
\hline
\end{tabular}

Figura 1. Representación gráfica del coeficiente de correlación-concordancia de Lin (CCC) y los límites de acuerdo del 95\% entre el volumen estimado y el volumen recolectado en la bolsa durante el parto vaginal (datos globales).
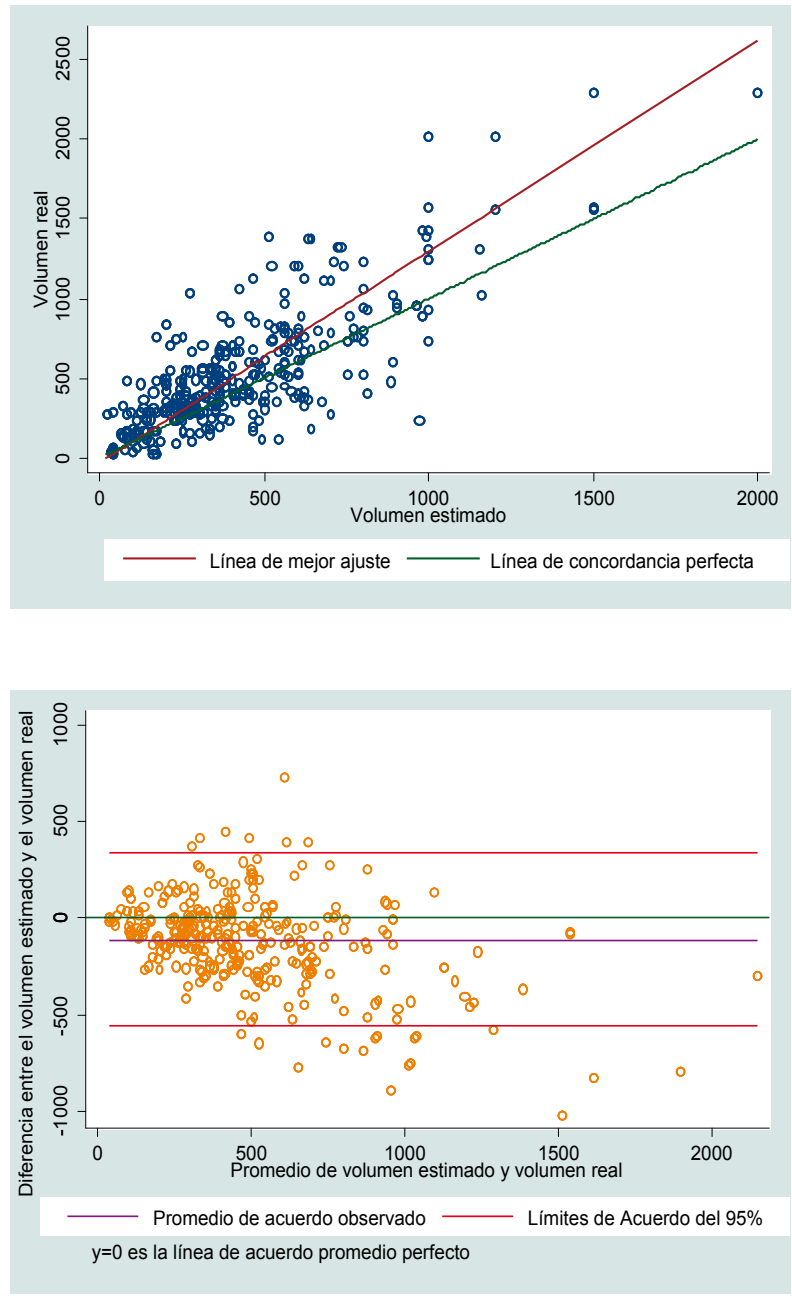

En los hospitales donde se realiza educación médica las decisiones sobre una intervención clínica deben recaer sobre el personal más calificado y con mayor experiencia. Por este motivo se evaluó la concordancia entre la estimación visual y el volumen real de sangrado según el papel desempeñado, el nivel de formación y los años de experiencia de quienes atendieron los partos.

El volumen recolectado en la bolsa durante el parto, en el presente estudio, es superior al reportado en estudios similares. Prasertcharoensuk ${ }^{10}$ reportó una media de sangrado intraparto de 383,81 mL. Tourné ${ }^{11}$ encontró una mediana de sangrado durante el parto de 190 mL y estimó el percentil 95 en 824 $\mathrm{mL}$. La mediana de volumen recolectado de 494 mL y el percentil 95 en $1.320 \mathrm{~mL}$, en este estudio, serían consecuencia de diferencias en la conducción y la atención del parto, así como del momento de aplicación de la bolsa recolectora y no debido a una mayor pérdida sanguínea durante el parto de las pacientes incluidas. Dada la técnica empleada en el presente estudio, es posible que haya caído líquido amniótico dentro de la bolsa recolectora, con una posible sobreestimación en el peso de la bolsa, sin que se hubiera podido establecer la magnitud de esta contaminación. Este hecho podría explicar la pobre correlación encontrada entre los cambios en la concentración de hemoglobina y el hematocrito con el volumen recolectado. La incidencia de hemorragia anormal del parto encontrada fue de 
Tabla 3. Correlación-concordancia de Lin para el volumen estimado y el volumen recolectado durante el parto vaginal.

\begin{tabular}{|c|c|c|c|c|c|c|}
\hline & $\mathbf{N}$ & $\rho$ (C) & IC $95 \% \rho$ (C) & $\rho$ Pearson's & Cs & Pendiente \\
\hline \multicolumn{7}{|c|}{ Papel desempeñado } \\
\hline Operador & 168 & 0,68 & $0,60-0,75$ & 0,75 & 0,91 & 1,34 \\
\hline Supervisor & 168 & 0,76 & $0,69-0,81$ & 0,83 & 0,91 & 1,32 \\
\hline \multicolumn{7}{|c|}{ Nivel de formación } \\
\hline Especialista & 55 & 0,71 & $0,58-0,80$ & 0,84 & 0,85 & 1,57 \\
\hline Residente & 164 & 0,76 & $0,69-0,82$ & 0,83 & 0,91 & 1,23 \\
\hline Estudiante & 117 & 0,66 & $0,56-0,75$ & 0,73 & 0,92 & 1,39 \\
\hline \multicolumn{7}{|l|}{ Experiencia } \\
\hline Mayor de 10 años & 26 & 0,73 & $0,50-0,86$ & 0,80 & 0,91 & 1,34 \\
\hline Hasta 10 años & 193 & 0,75 & $0,69-0,80$ & 0,83 & 0,90 & 1,30 \\
\hline Ninguna & 117 & 0,66 & $0,56-0,75$ & 0,73 & 0,92 & 1,39 \\
\hline
\end{tabular}

$\rho$ (C): coeficiente de correlación y concordancia.

Tabla 4. Diferencia promedio y límites de acuerdo de Bland y Altman entre el volumen estimado y el volumen recolectado durante el parto vaginal.

\begin{tabular}{|c|c|c|c|c|c|}
\hline & $\mathbf{N}$ & $\begin{array}{c}\text { Diferencia } \\
\text { promedio }(\mathrm{mL})\end{array}$ & $\begin{array}{l}\text { Límite inferior } \\
\text { 95\% (mL) }\end{array}$ & $\begin{array}{l}\text { Límite superior } \\
95 \%(\mathrm{~mL})\end{array}$ & $\begin{array}{l}\text { Amplitud de los } \\
\text { límites de acuerdo } \\
(\mathbf{m L})\end{array}$ \\
\hline \multicolumn{6}{|c|}{ Papel desempeñado } \\
\hline Operador & 168 & $-108,5$ & $-595,8$ & 378,8 & 974,6 \\
\hline Supervisor & 168 & $-111,8$ & $-526,7$ & 303,2 & 829,9 \\
\hline \multicolumn{6}{|c|}{ Nivel de formación } \\
\hline Especialista & 55 & $-112,4$ & $-539,0$ & 314,2 & 853,2 \\
\hline Residente & 164 & $-123,1$ & $-516,5$ & 270,4 & 786,8 \\
\hline Estudiante & 117 & $-91,0$ & $-625,1$ & 443,1 & $1.068,2$ \\
\hline \multicolumn{6}{|l|}{ Experiencia } \\
\hline Mayor de 10 años & 26 & $-88,8$ & $-459,0$ & 281,4 & 740,5 \\
\hline Hasta 10 años & 193 & $-124,6$ & $-529,9$ & 280,6 & 810,5 \\
\hline Ninguna & 117 & $-91,0$ & $-625,1$ & 443,1 & $1.068,2$ \\
\hline
\end{tabular}

Diferencia promedio $=$ volumen estimado - volumen real.

10,71\%. Este hallazgo coincide con la incidencia de hemorragia intraparto descrita en la literatura internacional, donde varía entre el 5,1 y el $27 \%{ }^{17-19,21,22}$ La incidencia de hemorragia intraparto severa (caída mayor del 10\% del hematocrito) de 5,36\% también es similar a la reportada en la literatura internacional ${ }^{25-27}$ y corresponde a los casos considerados como "near-misses" o morbilidad materna aguda severa.

Se observó que la incidencia varía según el criterio diagnóstico utilizado: si el diagnóstico se basa en los cambios de datos de laboratorio, la 
incidencia estará afectada por el hecho de que las maniobras exitosas para controlar la hemorragia posparto limitarían el descenso en las cifras del hematocrito.

Por otra parte, cerca de un 50\% de los casos de hemorragia posparto no fueron detectados clínicamente y el diagnóstico se hizo por el descenso del hematocrito mayor del 10\% durante el puerperio inmediato. El método diagnóstico con menor sensibilidad fue el criterio de transfusión sanguínea, ya que ninguna paciente del estudio fue transfundida por hemorragia aguda posparto.

El vacío de los programas de educación médica en pregrado y posgrado, en formación de la estimación del sangrado, se ha empezado a abordar mediante talleres en escenarios clínicos simulados para entrenar la estimación visual del sangrado intraoperatorio e intraparto. ${ }^{13,14}$

Los análisis de la distribución, dispersión y del promedio de la diferencia entre los métodos revelaron una subestimación creciente por el método visual al aumentar el volumen recolectado. La calidad de la estimación visual del sangrado está determinada por la experiencia clínica del observador, su nivel de formación, el conocimiento previo sobre la magnitud "normal" del sangrado intraparto y la capacitación específica en la estimación del volumen de sangrado. Bosé ${ }^{14}$ evaluó la capacidad de diferentes observadores para estimar la magnitud de sangrado en escenarios clínicos simulados por personal de salas de obstetricia y encontró una subestimación significativa en 5 de 12 escenarios, siendo mayor la subestimación en los escenarios con mayores sangrados simulados. El gradiente mayor de 1,0 de la línea de mejor ajuste confirma la creciente discrepancia entre el volumen estimado y el volumen calculado al incrementar la magnitud del mismo.

Los grupos con mayor permanencia dentro de las salas de parto (residentes y especialistas con experiencia menor de 10 años) muestran esta discrepancia en menor magnitud. Así mismo, este grupo tuvo mejor concordancia expresada por una mayor exactitud y precisión para realizar la estimación visual del sangrado que los observadores especialistas con más de 10 años de experiencia. Esta disminución en la confiabilidad podría deberse a una pérdida de entrenamiento como consecuencia de su mayor dedicación a las tareas propias del personal más experto (atención de las pacientes más complicadas), alejándolos de la atención de pacientes de bajo riesgo. Sin embargo, también se podría tratar de una subestimación del CCC debido al escaso número de evaluaciones realizadas por los observadores de esta categoría. ${ }^{17}$

La amplitud de los límites de acuerdo encontrados muestra que la estimación visual en la práctica clínica tiene una gran variabilidad y es muy poco precisa, por lo que se requeriría un método que permita graduar la cantidad recolectada dentro de bolsas o recipientes. El análisis gráfico de los mismos mostró que la dispersión de los datos creció a medida que aumentó el volumen recolectado en la bolsa, es decir, que la varianza del error no es constante (heterocedasticidad) y, por lo tanto, al incrementar el volumen recolectado durante la atención del parto, la estimación visual es menos confiable. Este hallazgo, que se presentó en todos los grupos de observadores analizados y en conjunto con la subestimación creciente encontrada por el gradiente del CCC, refuerza la necesidad de medir objetivamente la cantidad de sangrado intraparto.

Los resultados obtenidos en la presente investigación corroboran, mediante una aproximación cercana a la práctica diaria, los hallazgos mencionados por Prasertcharoensuk, ${ }^{10}$ Dildy $^{13} \mathrm{y}$ Bosé $^{14}$, y muestran la necesidad de desarrollar metodologías académicas de simulación que permitan el entrenamiento permanente para mejorar las habilidades y la calidad de la estimación visual y el desarrollo de alternativas de bajo costo que al mejorar la confiabilidad de la estimación del sangrado intraparto, permitan reducir la morbilidad severa y la mortalidad materna en el mundo. 


\section{REFERENCIAS}

1. Saboya MI, De La Hoz F. Tesis de grado: Mortalidad materna en Colombia. Una aproximación ecológica. Maestría en Salud Pública. Instituto de Salud Pública. Universidad Nacional de Colombia;2005.

2. Revised 1990. Estimates of maternal mortality: a new approach by WHO and Unicef. Geneva: WHO; 2000 .

3. Salazar A, Vásquez ML. Mortalidad materna en Cali ¿una década sin cambios? Colomb Med 1996;27: 117-24.

4. Peñuela AM, et al. Mortalidad materna y factores de riesgo. Estudio de casos y controles. Secretaría de Salud de Bogotá, Alcaldía Mayor; 1998.

5. Gil E. Mortalidad materna y factores de riesgo. Estudio de casos y controles. Área Metropolitana de Medellín, U. de Antioquia, Facultad Nacional de Salud Pública; 1989.

6. Vélez-Álvarez G, Gómez-Dávila JG, Zuleta-Tobón JJ. Análisis de las muertes maternas por hemorragia en el departamento de Antioquia, Colombia. Años 2004 y 2005. Rev Colomb Obstet Ginecol 2006;57: 147-55.

7. Pritchard JA. Changes in the blood volume during pregnancy and delivery. Anesthesiology 1965;26: 393-9.

8. Wallace G. Blood loss in obstetrics using a haemoglobin dilution technique. J Obstet Gynaecol Br Commonw 1967;74:64-7.

9. Razvi K, Chua S, Arulkumaran S, Ratnam SS. A comparison between visual estimation and laboratory determination of blood loss during the third stage of labour. Aust N Z J Obstet Gynaecol 1996;36: $152-4$.

10. Prasertcharoensuk W, Swadpanich U, Lumbiganon P. Accuracy of the blood loss estimation in the third stage of labor. Int J Gynaecol Obstet 2000;71:69-70.

11. Tourné G, Collet F, Lasnier P, Seffert P. Usefulness of a collecting bag for the diagnosis of post-partum hemorrhage. J Gynecol Obstet Biol Reprod (París) 2004;33:229-34.

12. República de Colombia. Ministerio de Protección Social. Guía de atención de las complicaciones hemorrágicas del embarazo; 1999.

13. Dildy GA 3rd, Paine AR, George NC, Velasco C. Estimating blood loss: can teaching significantly improve visual estimation? Obstet Gynecol 2004;104:601-6.
14. Bose P, Regan F, Paterson-Brown S. Improving the accuracy of estimated blood loss at obstetric haemorrhage using clinical reconstructions. BJOG 2006;113:919-24.

15. Lin LI. A concordance correlation coefficient to evaluate reproducibility. Biometrics 1989;45: 255-68.

16. Liao JJ, Lewis JW. A note on the concordance correlation coefficient. PDA J Pharm Sci Technol 2000;54:23-6.

17. Carrasco JL, Jover L, King TS, Chinchilli VM. Comparison of concordance correlation coefficient estimating approaches with skewed data. J Biopharm Stat. 2007;17:673-84.

18. Cepeda MS, Africano JM, Polo R, Alcalá R, Carr D. Agreement between percentage pain reductions calculated from numeric rating scores of pain intensity and those reported by patients with acute or cancer pain. Pain 2003;106:439-42.

19. Fleiss JL. The design and analysis of clinical experiments. New York: Wiley, 1986.

20. Bland JM, Altman DG. Statistical methods for assessing agreement between two methods of clinical measurement. Lancet 1986;1:307-10.

21. Bland JM, Altman DG. Comparing two methods of clinical measurement: a personal history. Int J Epidemiol 1995;24 Suppl 1:S7-14.

22. Sánchez R, Echeverry J. Validating scales used for measuring factors in medicine. Rev Salud Pública (Bogotá) 2004;6:302-18.

23. Norman GR, Streiner DL. Test of significance for ranked data. En: Norman GR, Streiner DL. Biostatistics: the bare essentials. 2nd edition. London: B.C. Decker 2000. p. 224-29.

24. WHO, Unicef, United Nations University. Iron deficiency anaemia assessment, prevention and control. A guide for programme managers. Geneva: WHO; 2001.

25. Henry A, Birch MR, Sullivan CA, Katz S, Wang YA. Primary postpartum haemorrhage in an Australian tertiary hospital: a case-control study. Aust N Z J Obstet Gynaecol 2005;45:233-6.

26. Magann EF, Evans S, Chauhan SP, Lanneau G, Fisk AD, Morrison JC. The length of the third stage of labor and the risk of postpartum hemorrhage. Obstet Gynecol 2005;105:290-3. 
27. Magann EF, Evans S, Hutchinson M, Collins R, Howard BC, Morrison JC. Postpartum hemorrhage after vaginal birth: an analysis of risk factors. South Med J 2005;98:419-22.

Conflicto de intereses: ninguno declarado.

Fuente de financiación: la presente investigación fue financiada por la vicerrectoría de investigaciones de la Universidad Nacional de Colombia, Sede Bogotá, Código DIB 20201007594. 